\title{
A note on "Optimal inventory model for items with imperfect quality and shortage backordering"
}

\author{
Jia-Tzer Hsu ${ }^{a}$ and Lie-Fern Hsu ${ }^{\text {b* }}$
}

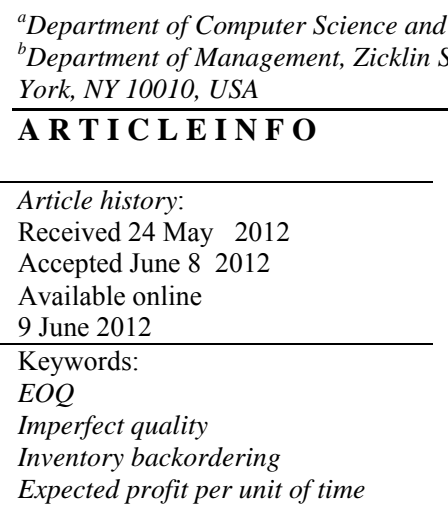

(C) 2012 Growing Science Ltd. All rights reserved

\section{Introduction}

Salameh and Jaber (2000) developed a model to determine the total profit per unit of time and the economic order quantity (EOQ) for a product purchased from a supplier. They assumed that each lot of the product received contained defective items with a known probability density function. Each order was subjected to a $100 \%$ screening process at a rate of $x$ units per unit time with $x>D$ (the annual demand). The defective items were sold at a discounted price at the end of the $100 \%$ screening process. Wee et al. (2007) extended the approach by Salameh and Jaber (2000) to consider permissible shortage backordering and the effect of varying backordering cost values. Maddah and Jaber (2008) identified a flaw in the work of Salameh and Jaber (2000) and proposed a new model that rectified the flaw using the renewal reward theorem. Chang and Ho (2010) revisited the work of Wee et al. (2007) and adopted the suggestion of Maddah and Jaber (2008) to use the renewal reward theorem to derive closed-form solutions for the optimal lot size and the maximum shortage level without using differential calculus. Since Salameh and Jaber (2000) assumed that the screening process and demand took place simultaneously, Wee et al. (2007) made the same assumption. However, in Wee et al.'s (2007) model, B units were backordered and then satisfied at the beginning of each cycle. They implicitly assumed that

* Corresponding author. Tel: 1-646-312-3656, Fax: 1-646-312-3620

E-mail: Lie-Fern.Hsu@baruch.cuny.edu (L.F. Hsu)

(C) 2012 Growing Science Ltd. All rights reserved. doi: $10.5267 / j$.ijiec.2012.05.007 
the $B$ units that were backordered were shipped to customers before the screening process (since they assumed that the maximum inventory level was the order size $y$ minus $B$ ). In other words, the $B$ units may have contained defective items. Moreover, if the $B$ backordered units were shipped to customers before the screening process, the time at which the defective items were sold should not have been $y / x$.

The purpose of this technical note is to point out the flaw in both Wee et al.'s (2007) and Chang and Ho's (2010) models and develop a corrected model. The rest of this paper is organized as follows: In section 2, we briefly review Wee et al.'s (2007) model and point out the flaw in the model. Then, in section 3 we develop a corrected model. Section 4 provides numerical examples to illustrate the differences between Wee et al.'s (2007) model and the corrected model.

\section{The model of Wee et al.}

The following notation and assumptions were used in Wee et al. (2007) and Chang and Ho (2010):

$\begin{array}{ll}y & \text { order size } \\ D & \text { the demand rate } \\ x & \text { the screening rate, } x>D \\ C & \text { the purchasing cost per unit } \\ K & \text { the ordering cost per order } \\ p & \text { the defective percentage in } y \\ f(p) & \text { the probability density function of } p \\ S & \text { the selling price per unit } \\ v & \text { the salvage value of per defective item, } v<c \\ d & \text { the screening cost per unit } \\ B & \text { the maximum backordering quantity in units } \\ b & \text { the backordering cost per unit per unit time } \\ h & \text { the holding cost per unit per unit time } \\ * & \text { the superscript representing optimal value }\end{array}$

Assumptions:

(1) The demand rate is known, constant, and continuous.

(2) The lead time is known and constant.

(3) The replenishment is instantaneous.

(4) The screening process and demand proceed simultaneously, but the screening rate is greater than demand rate, $x>D$.

(5) The defective items exist in lot size $y$. The defective percentage, $p$, has a uniform distribution with $[\alpha, \beta]$, where $0 \leq \alpha<\beta<1$.

(6) Shortage is completely backordered.

(7) A single product is considered. 


\section{Inventory Level}

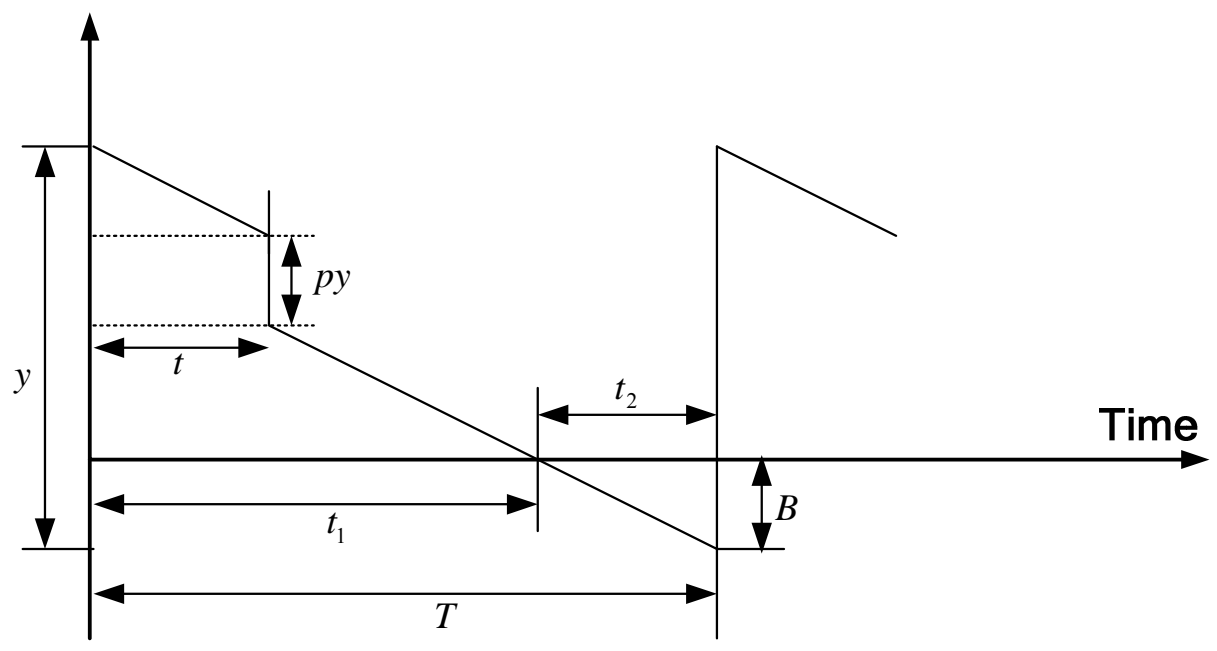

Fig.1. Inventory system with complete backordering (Wee et al.'s (2007) model).

The behavior of the inventory level is illustrated in Fig. 1, where $T$ is the cycle length, $p y$ is the number of defective items in each order, and $t$ is the total screening time of $y$ units ordered. Eqs. (1) - (8) were developed in Wee et al. (2007). To avoid shortages within the screening time $t, p$ is restricted to $p \leq 1-\frac{D}{x}$.

Let $T R(p, y)$ and $T C(B, y)$ be the total revenue and total cost per cycle; one has

$T R(p, y)=(1-p) y s+p y v$,

$T C(B, y)=K+c y+d y+h\left\{\frac{1}{2} \frac{(y-p y-B)^{2}}{D}+\frac{p y^{2}}{x}\right\}+\frac{1}{2} \frac{b B^{2}}{D}$.

The net profit per unit time, $T P U(B, y)$, is determined by the revenue per unit time less the average cost per unit time, which is

$T P U(B, y)=\frac{T R(p, y)-T C(B, y)}{T}$.

Since the replenishment cycle length $T=(1-p) y / D$, one has

$$
\begin{aligned}
\operatorname{TPU}(B, y)=D(s-v+ & \frac{h y}{x}+D\left(v-c-d-\frac{h y}{x}-\frac{K}{y}\right)\left(\frac{1}{1-p}\right) \\
& -\frac{h y(1-p)}{2}+h B-\frac{1}{2} \frac{h B^{2}}{(1-p) y}-\frac{1}{2} \frac{b B^{2}}{(1-p) y} .
\end{aligned}
$$


The expected value of $\operatorname{TPU}(B, y)$ is $\operatorname{ETPU}(B, y)$, where $\alpha \leq p \leq \beta$.

$$
\begin{aligned}
\operatorname{ETPU}(B, y)=D\left(s-v+\frac{h y}{x}\right)+\left(B-\frac{y}{2}\right) h+\frac{h y}{2} E[p] \\
+\left(D\left(v-c-d-\frac{h y}{x}-\frac{K}{y}\right)-\frac{(h+b) B^{2}}{2 y}\right) E\left[\frac{1}{1-p}\right] .
\end{aligned}
$$

By taking the first derivative of $\operatorname{ETPU}(B, y)$ with respect to $B$ and $y$, and setting the result to zero, one has

$$
\begin{aligned}
& \frac{\partial \operatorname{ETPU}(B, y)}{\partial B}=h-\left(\frac{1}{\beta-\alpha} \ln \left(\frac{1-\alpha}{1-\beta}\right)\right) B\left(\frac{h+b}{y}\right)=0 \\
& \frac{\partial \operatorname{ETPU}(B, y)}{\partial y}=\frac{h}{2}\left(\frac{\alpha+\beta}{2}-1\right)+\frac{D h}{x}+\left(D\left(\frac{K}{y^{2}}-\frac{h}{y}\right)+\frac{(h+b) B^{2}}{2 y}\right)\left(\frac{1}{\beta-\alpha} \ln \left(\frac{1-\alpha}{1-\beta}\right)\right)=0 .
\end{aligned}
$$

Wee et al. (2007) then suggested solving Eqs. (7) and (8) simultaneously for $B^{*}$ and $y^{*}$ using Maple. Note that both Wee et al. (2007) and Chang and Ho (2010) assumed that the maximum inventory level was $y-B$, and the total screening time for the $y$ units ordered was $y / x$. In other words, the $B$ items intended to satisfy the backorders were shipped to customers upon receipt of the new order before screening. After that, the screening process of the $y$ units ordered (including the $B$ units shipped to customers) began. The problem was that if $B$ units were shipped to customers in the beginning of each cycle, the $B$ units may have contained defective items, and the screening time should not have included the $B$ units shipped to customers. In that case, Wee et al. should have discussed what happened to the defective items contained in the $B$ units. If the $B$ units intended to satisfy the backorders were shipped to customers after screening, then the backordering period should have included the screening time for the satisfied backorders and the maximum inventory level in each cycle should have been $y$ instead of $y$ - $B$. Based on Wee et al.'s (2007) assumption that the screening process and demand proceed simultaneously, the correct behavior of the inventory system over time is depicted in Fig. 2 and the corrected mathematical model is developed as follows.

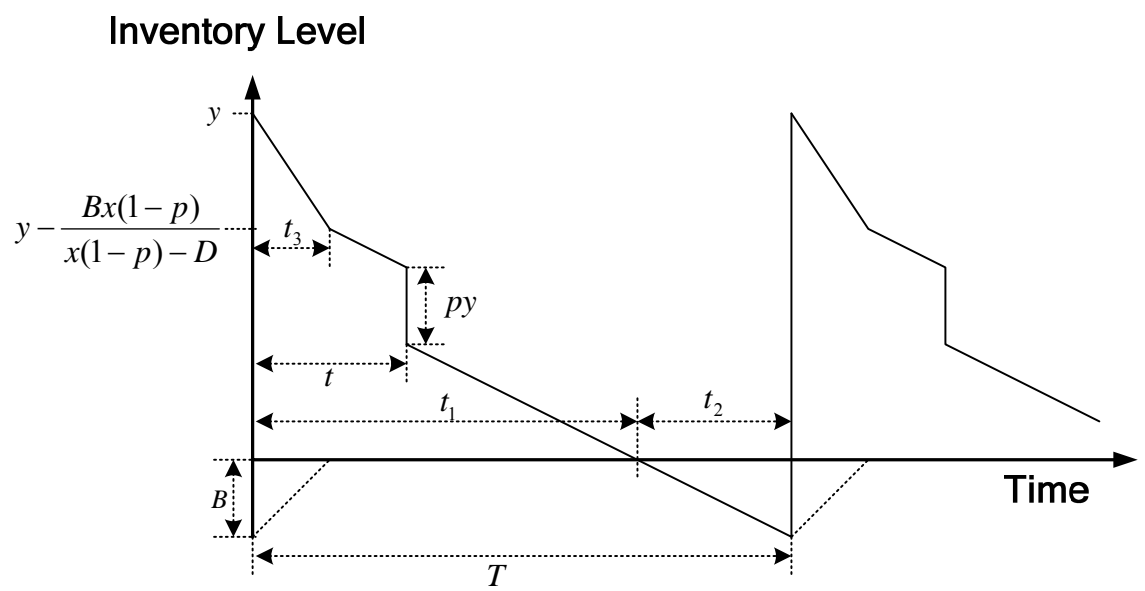

Fig. 2. Behavior of the inventory level over time for the corrected model 


\section{The corrected model}

In the beginning of each cycle, the inventory level begins with the order quantity $y$. The $B$ items intended to satisfy the backorders in each cycle will be filled at a rate of $x(1-p)-D$. After $t_{3}$, the time taken to fill $B$, the maximum shortage level per cycle, the inventory level will be reduced by $B+t_{3} D=x(1-p) t_{3}=\frac{B x(1-p)}{x(1-p)-D}$. Specifically, we have

$t_{1}=\frac{y(1-p)-B}{D}$

$t_{2}=\frac{B}{D}$

$t_{3}=\frac{B}{x(1-p)-D}$

The backordering cost per cycle is given as

$\frac{1}{2} b B\left(t_{2}+t_{3}\right)=\frac{1}{2} b B^{2}\left(\frac{1}{D}+\frac{1}{x(1-p)-D}\right)=\frac{1}{2} b B^{2}\left(\frac{1}{D}+\frac{1}{x(1-p-D / x)}\right)$,

and the holding cost per cycle is

$$
\begin{aligned}
& h\left\{\left(y(1-p)-\frac{1}{2} \frac{B x(1-p)}{(x(1-p)-D)}\right) t_{3}+\frac{1}{2}\left(y(1-p)-\frac{B x(1-p)}{(x(1-p)-D)}\right)\left(t_{1}-t_{3}\right)+\frac{p y^{2}}{x}\right\} \\
= & h\left\{\frac{1}{2} y(1-p) t_{3}+\frac{1}{2}\left(y(1-p)-\frac{B x(1-p)}{(x(1-p)-D)}\right) t_{1}+\frac{p y^{2}}{x}\right\} \\
= & h\left\{\frac{1}{2} y(1-p)\left(\frac{B}{x(1-p)-D}\right)+\frac{1}{2}\left(y(1-p)-\frac{B x(1-p)}{(x(1-p)-D)}\right)\left(\frac{y(1-p)-B}{D}\right)+\frac{p y^{2}}{x}\right\} \\
= & h\left\{\frac{1}{2} \frac{y B}{x}\left(\frac{(1-p)}{\left(1-p-\frac{D}{x}\right)}\right)+\frac{1}{2}\left(\frac{y^{2}(1-p)^{2}}{D}-\frac{y B(1-p)^{2}}{D\left(1-p-\frac{D}{x}\right)}-\frac{y B(1-p)}{D}+\frac{B^{2}(1-p)}{D\left(1-p-\frac{D}{x}\right)}\right)+\frac{p y^{2}}{x}\right\} .
\end{aligned}
$$

The total cost per cycle for this case is

$$
T C(B, y)=K+c y+d y
$$$$
+h\left\{\frac{1}{2} \frac{y B}{x}\left(\frac{(1-p)}{\left(1-p-\frac{D}{x}\right)}\right)+\frac{1}{2}\left(\frac{y^{2}(1-p)^{2}}{D}-\frac{y B(1-p)^{2}}{D\left(1-p-\frac{D}{x}\right)}-\frac{y B(1-p)}{D}+\frac{B^{2}(1-p)}{D\left(1-p-\frac{D}{x}\right)}\right)+\frac{p y^{2}}{x}\right\}
$$ 
$+\frac{1}{2} b B^{2}\left(\frac{1}{D}+\frac{1}{x(1-p-D / x)}\right)$,

and the expected profit per cycle is given by

$E[T P(B, y)]=E[T R(p, y)]-E[T C(B, y)]=(1-E[p]) y s+E[p] y v-K-c y-d y$

$-h \frac{1}{2} \frac{y B}{x} E\left[\frac{(1-p)}{\left(1-p-\frac{D}{x}\right)}\right]$

$-h \frac{1}{2}\left(\frac{y^{2} E\left[(1-p)^{2}\right]}{D}-\frac{y B}{D} E\left[\frac{(1-p)^{2}}{\left(1-p-\frac{D}{x}\right)}\right]-\frac{y B E[(1-p)]}{D}+\frac{B^{2}}{D} E\left[\frac{(1-p)}{\left(1-p-\frac{D}{x}\right)}\right]\right)$

$-h \frac{E[p] y^{2}}{x}-\frac{1}{2} b B^{2}\left(\frac{1}{D}+\frac{1}{x} E\left[\frac{1}{(1-p-D / x)}\right]\right)$.

Since the expected cycle time is

$$
E[T]=\frac{(1-E[p]) y}{D},
$$

using the renewal reward theorem, the expected profit per unit time is

$$
\begin{aligned}
& E T P U(B, y)=\frac{E[T P(B, y)]}{E[T]}=s D+v D \frac{E[p]}{(1-E[p])}-\frac{K D}{(1-E[p]) y}-\frac{c D}{(1-E[p])}-\frac{d D}{(1-E[p])} \\
& -\frac{1}{2} h \frac{D B}{x(1-E[p])} E\left[\frac{(1-p)}{\left(1-p-\frac{D}{x}\right)}\right] \\
& -\frac{1}{2} h\left(\frac{y E\left[(1-p)^{2}\right]}{(1-E[p])}-\frac{B}{(1-E[p])} E\left[\frac{(1-p)^{2}}{\left(1-p-\frac{D}{x}\right)}\right]-B+\frac{B^{2}}{y(1-E[p])} E\left[\frac{(1-p)}{\left(1-p-\frac{D}{x}\right)}\right]\right) \\
& -h \frac{E[p] y D}{x(1-E[p])}-\frac{1}{2} b B^{2}\left(\frac{1}{y(1-E[p])}+\frac{D}{x y(1-E[p])} E\left[\frac{1}{(1-p-D / x)}\right]\right) . \\
& \text { Let } A_{1}=E\left[\frac{(1-p)}{\left(1-p-\frac{D}{x}\right)}\right], A_{2}=E\left[\frac{(1-p)^{2}}{\left(1-p-\frac{D}{x}\right)}\right], \text { and } A_{3}=E\left[\frac{1}{\left(1-p-\frac{D}{x}\right)}\right],
\end{aligned}
$$


by taking the first derivative of $\operatorname{ETPU}(B, y)$ with respect to $B$ and $y$, we have

$$
\begin{aligned}
& \frac{\partial E T P U(B, y)}{\partial B}=h\left\{-\frac{1}{2} \frac{D A_{1}}{x(1-E[p])}+\frac{1}{2} \frac{A_{2}}{(1-E[p])}+\frac{1}{2}-\frac{B A_{1}}{y(1-E[p])}\right\} \\
& -b B\left(\frac{1}{y(1-E[p])}+\frac{D A_{3}}{x y(1-E[p])}\right), \\
& \frac{\partial E T P U(B, y)}{\partial y}=\frac{K D}{(1-E[p]) y^{2}}-h\left(\frac{1}{2} \frac{E\left[(1-p)^{2}\right]}{(1-E[p])}-\frac{B^{2} A_{1}}{2 y^{2}(1-E[p])}+\frac{E[p] D}{x(1-E[p])}\right) \\
& +\frac{1}{2} b B^{2}\left(\frac{1}{y^{2}(1-E[p])}+\frac{D A_{3}}{x y^{2}(1-E[p])}\right) .
\end{aligned}
$$

Taking the second derivative, we have

$$
\frac{\partial^{2} \operatorname{ETPU}(B, y)}{\partial B^{2}}=-\frac{h A_{1}}{y(1-E[p])}-b\left(\frac{1}{y(1-E[p])}+\frac{D A_{3}}{x y(1-E[p])}\right),
$$

$\frac{\partial^{2} \operatorname{ETPU}(B, y)}{\partial y^{2}}=-\frac{2 K D}{y^{3}(1-E[p])}-\frac{h B^{2} A_{1}}{y^{3}(1-E[p])}-b B^{2}\left(\frac{1}{y^{3}(1-E[p])}+\frac{D A_{3}}{x y^{3}(1-E[p])}\right)$,

$\frac{\partial^{2} \operatorname{ETPU}(B, y)}{\partial B \partial y}=\frac{B h A_{1}}{y^{2}(1-E[p])}+b B\left(\frac{1}{y^{2}(1-E[p])}+\frac{D A_{3}}{x y^{2}(1-E[p])}\right)$,

and

$$
\left(\frac{\partial^{2} E T P U(B, y)}{\partial B^{2}}\right)\left(\frac{\partial^{2} E T P U(B, y)}{\partial y^{2}}\right)-\left(\frac{\partial^{2} E T P U(B, y)}{\partial B \partial y}\right)^{2}=\frac{2 K D\left(h A_{1}+b+b A_{3} D / x\right)}{y^{4}(1-E[p])^{2}} .
$$

Note that if the value of $p<1-\frac{D}{x}$, we have $\frac{\partial^{2} \operatorname{ETPU}(B, y)}{\partial B^{2}}<0, \frac{\partial^{2} \operatorname{ETPU}(B, y)}{\partial y^{2}}<0$, and $\left(\frac{\partial^{2} E T P U(B, y)}{\partial B^{2}}\right)\left(\frac{\partial^{2} E T P U(B, y)}{\partial y^{2}}\right)-\left(\frac{\partial^{2} E T P U(B, y)}{\partial B \partial y}\right)^{2}>0$, which implies that there exist unique solutions of $B$ and $y$ that maximize the expected profit per unit time and are given as

$$
y^{*}=\sqrt{\frac{2 K D}{h\left\{E\left[(1-p)^{2}\right]-R^{2} A_{1}+2 E[p] \frac{D}{x}\right\}-b R^{2}\left(1+A_{3} \frac{D}{x}\right)}},
$$

$B^{*}=y^{*} R$, 
With

$R=\frac{h\left(1-E[p]-A_{1} D / x+A_{2}\right)}{2\left(h A_{1}+b+b A_{3} D / x\right)}$.

\section{Numerical examples}

For the purpose of comparison, we apply the same parameters as in Wee et al. (2007):

Demand rate, $D$

Ordering cost, $K$

Holding cost, $h$

Screening rate, $x$

Screening cost, $d$

Purchase cost, $c$

Backordering cost, $b$

Selling price of good quality items, $s$

The salvage value of defective items, $v$
$=50,000$ units/year,

$=100 /$ cycle,

$=\$ 5 /$ unit $/$ year

$=1 \mathrm{unit} / \mathrm{min}=175,200$ units/year

$=\$ 0.5 /$ unit

$=\$ 25 /$ unit

$=\$ 10 /$ unit/year

$=\$ 50 /$ unit

$=\$ 20 /$ unit

If the defective percentage follows a uniform distribution with

$$
f(p)=\left\{\begin{array}{l}
\frac{1}{\beta}, 0 \leq p \leq \beta \\
0, \text { otherwise }
\end{array}\right.
$$

then we have

$$
\begin{aligned}
& E[p]=\int_{0}^{\beta} p f(p) d p=\int_{0}^{\beta} \frac{p}{\beta} d p=\frac{\beta}{2}, \quad E\left[(1-p)^{2}\right]=\int_{0}^{\beta}(1-p)^{2} f(p) d p=\int_{0}^{\beta} \frac{(1-p)^{2}}{\beta} d p=1-\beta+\frac{\beta^{2}}{3}, \\
& A_{1}=E\left[\frac{(1-p)}{\left(1-p-\frac{D}{x}\right)}\right]=\int_{0}^{\beta} \frac{1-p}{1-p-D / x} f(p) d p=\int_{0}^{\beta} \frac{1}{\beta}\left[1+\frac{D / x}{1-p-D / x}\right] d p=1+\frac{D}{\beta x} \ln \left(\frac{1-D / x}{1-D / x-\beta}\right), \\
& A_{2}=E\left[\frac{(1-p)^{2}}{\left(1-p-\frac{D}{x}\right)}\right]=\int_{1-D / x-\beta}^{1-D / x} \frac{(z+D / x)^{2}}{Z} f(z) d z=\int_{1-D / x-\beta}^{1-D} \frac{1}{\beta}\left(z+2 D / x+\frac{(D / x)^{2}}{Z}\right) d z \\
& =1-\frac{D}{x}-\frac{\beta}{2}+2 \frac{D}{x}+\frac{1}{\beta}\left(\frac{D}{x}\right)^{2} \ln \left(\frac{1-D / x}{1-D / x-\beta}\right)=1+\frac{D}{x}-\frac{\beta}{2}+\frac{1}{\beta}\left(\frac{D}{x}\right)^{2} \ln \left(\frac{1-D / x}{1-D / x-\beta}\right),
\end{aligned}
$$


$A_{3}=E\left[\frac{1}{\left(1-p-\frac{D}{x}\right)}\right]=\frac{1}{\beta} \ln \left(\frac{1-D / x}{1-D / x-\beta}\right)$.

Specifically, if $\beta=0.04$, we obtain the results given in Tables 1-4. Numerical examples show that in terms of the two decision variables (order size $y$ and the maximum backordering quantity in units $B$ ), there is a significant difference between the corrected model and Wee et al.'s (2007) model. The results also show that that the penalty of using Wee et al.'s (2007) model can be significant when the screening rate $x$ is small.

Table 1

Numerical results, $p$ is uniformly distributed between 0 and $0.04, D=50,000, K=100, h=5, b=10$.

\begin{tabular}{lllll}
\hline$x$ & 75,000 & 125,000 & 150,000 & 175,200 \\
\hline Corrected $B^{*}$ & 156.87 & 308.13 & 349.03 & 379.32 \\
Corrected $y^{*}$ & $1,503.34$ & $1,594.05$ & $1,619.40$ & $1,638.40$ \\
$\operatorname{ETPU}\left(B^{*}, y^{*}\right)$ & $1,212,600.16$ & $1,212,986.38$ & $1,213,086.60$ & $1,213,159.67$ \\
$B^{\text {Wee }}\left(\right.$ Wee et al.'s $\left.B^{*}\right)$ & 565.63 & 570.21 & 571.37 & 572.21 \\
$y^{\text {Wee }}\left(\right.$ Wee et al.'s $\left.y^{*}\right)$ & $1,731.53$ & $1,745.54$ & $1,749.10$ & $1,751.67$ \\
$\operatorname{ETPU}\left(B^{\text {Wee }}, y^{\text {Wee }}\right)$ & $1,210,481.87$ & $1,212,558.45$ & $1,212,817.31$ & $1,212,974.50$ \\
$\operatorname{ETPU}\left(B^{*}, y^{*}\right)$ & $2,118.29$ & 427.93 & 269.29 & 185.17 \\
$-\operatorname{ETPU}\left(B^{\text {Wee }}, y^{\text {Wee }}\right)$ & & & & \\
\hline
\end{tabular}

\section{Table 2}

Numerical results, $p$ is uniformly distributed between 0 and $\beta, D=50,000, x=175,200, K=100, h=5, b=10$

\begin{tabular}{lllllllll}
\hline$\beta$ & 0.04 & 0.06 & 0.08 & 0.10 & 0.20 & 0.30 & 0.40 & 0.50 \\
\hline Corrected $B^{*}$ & 379.32 & 375.86 & 372.29 & 368.63 & 348.68 & 325.69 & 299.01 \\
Corrected $y^{*}$ & $1,638.40$ & $1,647.32$ & $1,656.16$ & $1,664.90$ & $1,706.79$ & $1,744.81$ & $1,777.62$ & $1,803.55$ \\
$\operatorname{ETPU}\left(B^{*}, y^{*}\right)$ & $1,213,159.67$ & $1,210,236.65$ & $1,207,252.02$ & $1,204,203.81$ & $1,187,934.48$ & $1,169,727.90$ & $1,149,218.14$ & $1,125,940.50$ \\
$B^{\text {Wee }}\left(\right.$ Wee et al.'s $\left.B^{*}\right)$ & 572.21 & 569.50 & 566.676 & 563.75 & 547.535 & 528.52 & 506.60 & 481.78 \\
$y^{\text {Wee }}\left(\right.$ Wee et al.'s $\left.y^{*}\right)$ & $1,751.67$ & $1,761.33$ & $1,770.86$ & $1,780.27$ & $1,825.12$ & $1,865.36$ & $1,899.76$ & $1,927.13$ \\
$\operatorname{ETPU}\left(B^{\text {Wee }}, y^{\text {Wee }}\right)$ & $1,212,974.50$ & $1,210,047.83$ & $1,207,059.39$ & $1,204,007.22$ & $1,187,715.25$ & $1,169,479.38$ & $1,148,928.90$ & $1,125,587.61$ \\
$\operatorname{ETPU}\left(B^{*}, y^{*}\right)$ & 185.17 & 188.82 & 192.63 & 196.59 & 219.23 & 248.52 & 289.24 & 352.89 \\
$-\operatorname{ETPU}\left(B^{\text {Wee }}, y^{\text {Wee }}\right)$ & & & & & & & & \\
\hline
\end{tabular}

Table 3

Numerical results for different holding cost, $p$ is uniformly distributed between 0 and $0.04, D=50,000, x=175,200, K=100, b=10$

\begin{tabular}{llllll}
\hline$h$ & 1 & 3 & 5 & 8 & 10 \\
\hline Corrected $B^{*}$ & 209.30 & 324.18 & 379.32 & 421.82 & 436.97 \\
Corrected $y^{*}$ & $3,314.84$ & $2,022.53$ & $1,638.40$ & $1,366.48$ & $1,258.27$ \\
$\operatorname{ETPU}\left(B^{*}, y^{*}\right)$ & $1,216,309.45$ & $1,214,342.54$ & $1,213,159.67$ & $1,211,920.31$ & $1,211,278.13$ \\
$B^{\text {Wee }}\left(\right.$ Wee et al.'s $\left.B^{*}\right)$ & 299.54 & 476.67 & 572.21 & 659.57 & 698.75 \\
$y^{\text {Wee }}\left(\right.$ Wee et al.'s $\left.y^{*}\right)$ & $3,362.15$ & $2,107.74$ & $1,751.67$ & $1,514.31$ & $1,426.03$ \\
$\operatorname{ETPU}\left(B^{\text {Wee }}, y^{\text {Wee }}\right)$ & $1,216,291.22$ & $1,214,252.66$ & $1,212,974.50$ & $1,211,565.07$ & $1,210,796.32$ \\
$\operatorname{ETPU}\left(B^{*}, y^{*}\right)$ & 18.23 & 89.88 & 185.17 & 355.24 & 481.81 \\
$-\operatorname{ETPU}\left(B^{\text {Wee }}, y^{\text {Wee }}\right)$ & & & & & \\
\hline
\end{tabular}




\section{Table 4}

Numerical results for different backordering cost, $p$ is uniformly distributed between 0 and 0.04 , $D=50,000, x=175,200, K=100, h=5$

\begin{tabular}{llllll}
\hline$b$ & 5 & 10 & 15 & 20 & $\infty$ \\
\hline Corrected $B^{*}$ & 617.97 & 379.32 & 274.24 & 214.88 & 0 \\
Corrected $y^{*}$ & $1,779.46$ & $1,638.40$ & $1,579.38$ & $1,546.89$ & $1,434.48$ \\
$\operatorname{ETPU}\left(B^{*}, y^{*}\right)$ & $1,213,653.39$ & $1,213,159.67$ & $1,212,926.94$ & $1,212,791.24$ & $1,212,274.30$ \\
$B^{\text {Wee }}\left(\right.$ Wee et al.'s $\left.B^{*}\right)$ & 988.187 & 572.21 & 405.01 & 313.88 & 0 \\
$y^{\text {Wee }}\left(\right.$ Wee et al.'s $\left.y^{*}\right)$ & $2,016.71$ & $1,751.67$ & $1,653.119$ & $1,601.42$ & $1,434.48$ \\
$\operatorname{ETPU}\left(B^{\text {Wee }}, y^{\text {Wee }}\right)$ & $1,213,312.69$ & $1,212,974.50$ & $1,212,799.01$ & $1,212,693.36$ & $1,212,274.30$ \\
$\operatorname{ETPU}\left(B^{*}, y^{*}\right)$ & 340.70 & 185.17 & 127.93 & 97.88 & 0 \\
$-\operatorname{ETPU}\left(B^{\text {Wee }}, y^{\text {Wee }}\right)$ & & & & & \\
\hline
\end{tabular}

\section{References}

Chang, H.C., \& Ho, C.H. (2010). Exact closed-form solutions for "optimal inventory model for items with imperfect quality and shortage backordering. Omega, 38, 233-237.

Maddah, B., \& Jaber, M.Y. (2008). Economic order quantity for items with imperfect quality: Revisited. International Journal of Production Economics, 112, 808-815.

Salameh, M.K., \& Jaber, M.Y. (2000) Economic production quantity model for items with imperfect quality. International Journal of Production Economics, 64, 59-64.

Wee, H.M., Yu, J., \&. Chen, M.C. (2007). Optimal inventory model for items with imperfect quality and shortage backordering, Omega, 35, 7-11. 